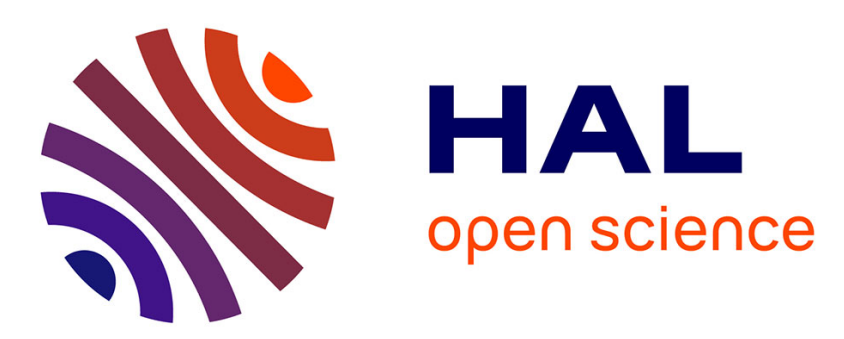

\title{
Présentation du logiciel de simulation bidimensionnelle de technologie silicium - MOBIDIC
}

D. Collard, I. Desoutter, M. Lobet, J.N. Decarpigny

\section{To cite this version:}

D. Collard, I. Desoutter, M. Lobet, J.N. Decarpigny. Présentation du logiciel de simulation bidimensionnelle de technologie silicium - MOBIDIC. Revue de Physique Appliquée, 1986, 21 (1), pp.35-44. 10.1051/rphysap:0198600210103500 . jpa-00245408

\section{HAL Id: jpa-00245408 https://hal.science/jpa-00245408}

Submitted on 1 Jan 1986

HAL is a multi-disciplinary open access archive for the deposit and dissemination of scientific research documents, whether they are published or not. The documents may come from teaching and research institutions in France or abroad, or from public or private research centers.
L'archive ouverte pluridisciplinaire HAL, est destinée au dépôt et à la diffusion de documents scientifiques de niveau recherche, publiés ou non, émanant des établissements d'enseignement et de recherche français ou étrangers, des laboratoires publics ou privés. 


\title{
Présentation du logiciel de simulation bidimensionnelle de technologie silicium - MOBIDIC $\left(^{+}\right)$
}

\author{
D. Collard $\left({ }^{*}\right)$, I. Desoutter $\left({ }^{* *}\right)$, M. Lobet $\left({ }^{* * *}\right)$ et J. N. Decarpigny $\left({ }^{*}\right)$ \\ (*) I.S.E.N., Institut Supérieur d'Electronique du Nord, 3, rue François Baës, 59046 Lille Cedex, France \\ (**) Matra-Harris Semiconducteurs, B.P. no 942, 44075 Nantes Cedex, France \\ $\left({ }^{* * *}\right)$ Laboratoire de Microélectronique, Bât. Maxwell, 3, Place du Levant, B-1348 Louvain-la-Neuve, Belgique
}

(Reçu le 25 novembre 1984, révisé les 24 juillet et 23 septembre 1985, accepté le 24 septembre 1985)

\begin{abstract}
Résumé. - Un programme de simulation bidimensionnelle de technologie silicium, MOBIDIC, a été développé. Ce programme permet la simulation de l'implantation ionique à travers un masque quelconque et la résolution des équations du couplage entre impuretés sous ambiance inerte ou oxydante. Pour le bore et l'arsenic, le modèle de diffusion inclut l'effet du champ électrique, des lacunes chargées et la formation d'agglomérats. La diffusion du phosphore est basée sur le modèle de Fair. La croissance bidimensionnelle d'oxyde est donnée par une loi analytique les équations de diffusion sont alors intégrées dans un domaine à géométrie variable. Le programme utilise la méthode des éléments finis, les équations temporelles étant résolues par un schéma implicite incomplet. Les résultats de la simulation de technologie peuvent être introduits dans le simulateur de dispositif MINIMOS afin de déterminer le comportement électrique des structures actives. Ce couplage technologie/dispositif est présenté dans le cas d'une technologie n-MOS 6 microns.

Abstract. - A two dimensional process simulator, MOBIDIC, has been developed. This program allows the simulation of ion implantation through arbitrary masks and the resolution of the diffusion equations taking account of the interaction between impurities under inert or oxidizing ambient. For boron and arsenic, the diffusion model includes electric field, charged vacancies and clustering effects. The phosphorus diffusion is based upon Fair's model. The two dimensional oxide growth is given by an analytical formula, the diffusion equations being solved in an evolutive domain. The program uses the finite element method, the temporal equations being solved by an incomplete implicit scheme. The results of the process simulations can be introduced in the device simulator MINIMOS to determine the electrical behaviour of the components. This process-device connection is presented in the case of a 6-microns n-MOS technology.
\end{abstract}

\section{Introduction.}

La connaissance actuelle des matériaux utilisés en technologie silicium, ainsi que les récents progrès réalisés dans les techniques de lithogravure permettent l'intégration à grande échelle de composants microniques et laissent déjà entrevoir le domaine submicronique. Parallèlement à cet essor, la mise au point des séquences technologiques est de complexité croissante, la réduction des dimensions faisant intervenir de manière critique de nombreux effets souvent couplés. De plus, la structure interne des composants est maintenant typiquement bidimensionnelle dans la mesure où leur extension latérale est du même ordre de grandeur que l'extension en profondeur de leur zone

$\left({ }^{+}\right)$Communication présentée aux Journées du G.C.I.S., Toulouse les 15 et 16 décembre 1983 . active. Ces raisons, étroitement liées, motivent le développement de logiciels de simulation bidimensionnelle de technologie. Ces programmes doivent calculer, selon les conditions expérimentales, les profils de dopage et les épaisseurs d'oxyde, non seulement dans la direction perpendiculaire à la surface de la structure [1], mais dans tout le domaine bidimensionnel afin de prévoir les effets latéraux. Ces résultats permettent une description réelle du transistor qui peut alors être introduit dans un simulateur de dispositif $[2,3]$ afin d'avoir directement accès à des informations électriques.

Plusieurs codes de simulation bidimensionnelle de technologie sont en développement [4-10], qui se différencient essentiellement par les approches numériques qu'ils utilisent.

Un programme de type recherche, MOBIDIC, a été élaboré. Utilisant la méthode des éléments finis, ce 
programme permet, grâce à la souplesse de sa structure, de simuler des enchaînements technologiques complexes composés des étapes élémentaires suivantes :

- implantation ionique sous masque quelconque (arsenic, bore et phosphore),

- prédépôt thermique (arsenic, bore et phosphore),

- recuits thermiques en ambiance inerte ou oxydante $\left(\mathrm{H}_{2} \mathrm{O}, \mathrm{O}_{2}\right.$, vapeur),

- dépôt et attaque d'oxyde à basse ou haute température.

De plus, de nouveaux modèles physiques peuvent être inclus et testés. Les résultats de ce programme sont, soit directement exploités de manière graphique, soit introduits dans le simulateur de dispositif MINIMOS [2].

Dans un premier temps, les modèles physiques intégrés dans le programme sont présentés. Ensuite, la partie numérique, volontairement brève, précise les algorithmes qui traitent ces différents modèles. Enfin, un exemple d'application du programme est présenté, dans le cas d'une technologie n-MOS.

\section{Les modèles physiques.}

2.1 ImPLANTATION IONIQUE. - Les profils implantés sont calculés par une méthode semi-analytique qui permet de prendre en compte la topologie réelle du masque [11]. En se référant à la structure homogène illustrée en figure 1, la probabilité pour qu'un ion, percutant la surface du silicium en $\left(x_{1}, y_{1}\right)$, se loge en $(x, y)$ est donnée par :

$$
\begin{aligned}
f(x, y)= & \frac{1}{2 \pi \cdot \Delta X \cdot \Delta R_{\mathrm{p}}} \times \\
& \times \exp \left[-\frac{\left(x-x_{1}\right)^{2}}{2 . \Delta X^{2}}-\frac{\left(y-y_{1}+R_{\mathrm{p}}\right)^{2}}{2 . \Delta R_{\mathrm{p}}^{2}}\right]
\end{aligned}
$$

$R_{\mathrm{p}}, \Delta R_{\mathrm{p}}, \Delta X$ sont respectivement la pénétration moyenne, l'écart type et la dispersion latérale des ions implantés dans le silicium [12]. Le profil résultant est

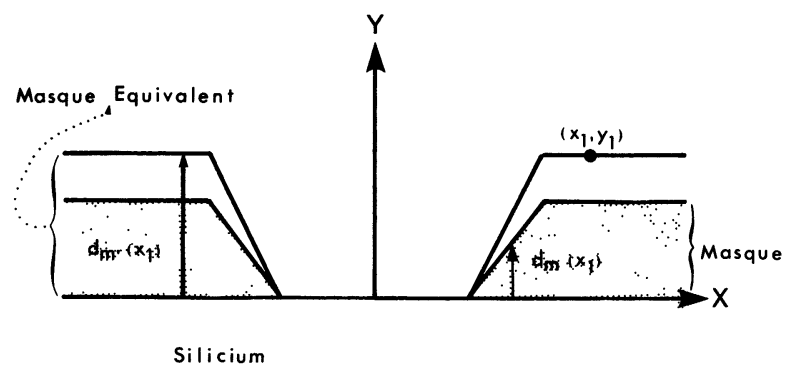

Fig. 1. - Représentation schématique du masque d'implantation (masque : zone hachurée).

[Schematic representation on the implantation mask (Mask : dashed area).] alors obtenu par une intégrale de surface de la densité de probabilité $f$ si l'on considère le masque comme une surépaisseur de silicium. Cette substitution doit être équivalente au sens du pouvoir d'arrêt des différents matériaux :

$$
d_{\mathrm{m}^{\prime}}\left(x_{1}\right)=d_{\mathrm{m}}\left(x_{1}\right) \cdot \frac{R_{\mathrm{p}}}{R_{\mathrm{p}_{\mathrm{m}}}}
$$

$R_{\mathrm{p}_{\mathrm{m}}}$ étant la pénétration des ions dans le matériau constitutif du masque, $d_{\mathrm{m}}$ l'épaisseur du masque et $d_{\mathrm{m}}^{\prime}$ l'épaisseur équivalente de silicium.

Les équations (1) et (2) conduisent à l'expression du profil implanté dans la structure de la figure 1 :

$$
\begin{aligned}
& F(x, y) \equiv \frac{N_{\square}}{2 \pi \cdot \Delta X . \Delta R_{\mathrm{p}}} \\
& \int_{-\infty}^{+\infty} \exp \left[-\frac{\left(x-x_{1}\right)^{2}}{2 . \Delta X^{2}}-\frac{\left(y-d_{\mathrm{m}}^{\prime}\left(x_{1}\right)+R_{\mathrm{p}}\right)^{2}}{2 . \Delta R_{\mathrm{p}}^{2}}\right] \mathrm{d} x_{1}
\end{aligned}
$$

$y \leqslant 0$

où $N_{\square}$ est la dose implantée.

Le résultat est obtenu par intégration numérique.

Cette méthode permet de déterminer pour les structures auto-alignées l'influence de la topologie du masque sur la pénétration latérale des dopants avant recuit. La figure 2 montre par exemple le rôle du profil d'attaque du poly-silicium sur le dopage source/drain d'une technologie n-MOS. Cette méthode semi-analytique a ici été développée pour une distribution de type gaussien. Pour le bore et l'arsenic, d'autres distributions

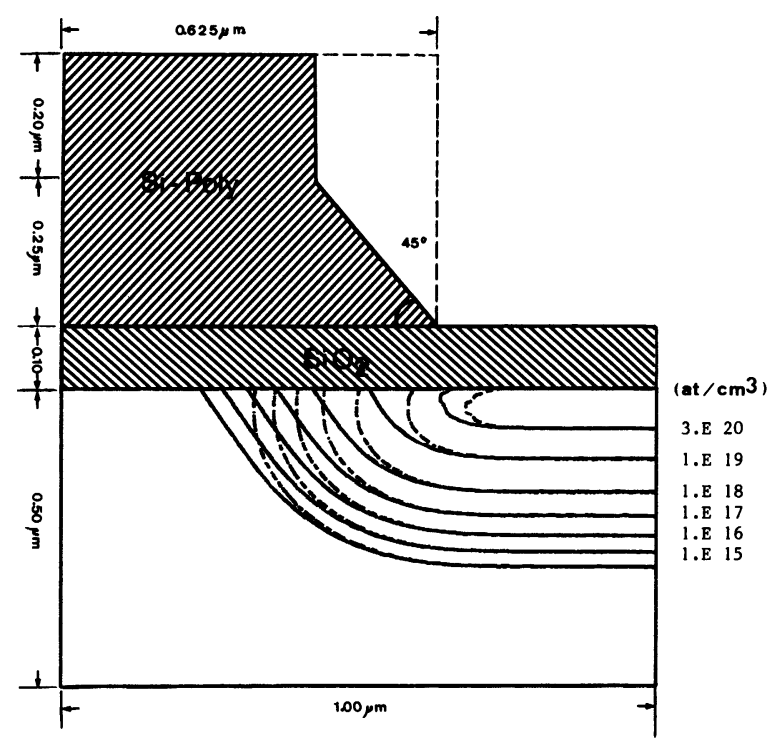

Fig. 2. - Implantation de phosphore : $N_{\mathrm{o}}=5 \times$ $10^{15} \mathrm{~cm}^{-2}, 120 \mathrm{keV}$. Traits pleins : masque réel. Traits pointillés : hypothèse du masque abrupt. Isovaleurs en $\mathrm{cm}^{-3}$.

[Phosphorus implantation: $N=5 \times 10^{15} \mathrm{~cm}^{-2}, 120 \mathrm{keV}$. Full lines : real mask edge. Dashed lines : hypothetical infinite thick screen. Isovalues in $\mathrm{cm}^{-3}$.] 
ont été proposées $[1,13,14]$ qui s'appuient sur de nombreux résultats expérimentaux. Ces distributions d'ordre supérieur seront incluses dans le programme MOBIDIC; ainsi pour l'arsenic, les profils implantés sont calculés à partir de distribution de type bi-gaussien [13] afin de prendre en compte la dissymétrie des profils, tandis que pour le bore, une distribution de type Pearson IV [15] a été choisie. La prise en compte de ces distributions se réalise au niveau de l'équation (3).

2.2 DIFFUSION DES IMPURETÉS III-V DANS LE SILICIUM. - Les modèles utilisés pour la diffusion des impuretés sont obtenus par une extension, à deux dimensions, des équations de base établies pour les simulations unidimensionnelles [1].

La redistribution du bore et de l'arsenic est régie par l'équation de diffusion suivante [4] :

$$
\begin{aligned}
\frac{\partial C}{\partial t} & =\frac{\partial}{\partial x}\left(D \frac{\partial N}{\partial x}\right)+\frac{\partial}{\partial y}\left(D \frac{\partial N}{\partial y}\right) \pm \\
& \pm\left[\frac{\partial}{\partial x}\left(D \cdot N \cdot \frac{\partial \psi}{\partial x}\right)+\frac{\partial}{\partial y}\left(D \cdot N \cdot \frac{\partial \psi}{\partial y}\right)\right] .
\end{aligned}
$$

dans laquelle le terme de conduction rend compte de l'effet du champ électrique auto-induit, le signe dépendant de l'état de charge du dopant (+ pour l'arsenic, - pour le bore). Dans cette équation $C$ et $N$ sont respectivement la concentration totale et active de l'impureté considérée et $D$ son coefficient de diffusion effectif. $\psi$ est le potentiel réduit qui est calculé en fonction de la concentration en électrons libres $n$, et de la concentration intrinsèque $n_{\mathrm{i}}$ :

$$
\psi=\log \frac{n}{n_{\mathbf{i}}} .
$$

L'équation (4) est écrite dans l'hypothèse où seuls les atomes électriquement actifs diffusent. Elle permet la prise en compte de l'accélération de la diffusion due aux lacunes chargées par l'expression du coefficient de diffusion effectif $[16,17]$ :

$$
D=D_{\mathrm{i}} \frac{1+\beta f}{1+\beta}
$$

dans lequel : $f=\frac{n}{n_{\mathrm{i}}}, \beta=100$ pour l'arsenic

$$
f=\frac{n_{\mathrm{i}}}{n}, \beta=19 \text { pour le bore. }
$$

et $D_{\mathrm{i}}$ est le coefficient de diffusion intrinsèque.

Dans le cas de forts dopages (régions de source/ drain de transistor MOS ou émetteurs en technologie bipolaire par exemple), la diffusion est fortement non linéaire. Cela est dû non seulement à l'effet des lacunes chargées, équation (6), mais également à d'autres effets tels que la limite de solubilité et la formation d'agglomérats. Ces derniers effets, actuellement négligés pour le bore, sont simulés conformément à un modèle récent [18], dans lequel le mécanisme de formation d'agglomérat d'arsenic est résumé par la relation suivante :

$$
3 \mathrm{As}^{+}+\mathrm{e}^{-} \rightleftharpoons \mathrm{As}_{3}^{2+} .
$$

La loi d'action de masse conduit à une relation entre les concentrations totale $C_{\mathrm{As}}$ et active $N_{\mathrm{As}}$ :

$$
C_{\mathrm{As}}=N_{\mathrm{As}}\left[1+\frac{3 / 2\left(N_{\mathrm{As}} / N_{\max }\right)^{3}}{\left(1-N_{\mathrm{As}} / N_{\max }\right)^{3}}\right]
$$

dans lequel $N_{\max }$ s'identifie à la limite de solubilité de l'arsenic substitutionnel et dépend de la température.

Les simulations sont effectuées dans l'hypothèse de neutralité électrique locale. La concentration en électrons libres s'exprime alors en fonction des diverses concentrations présentes dans le substrat par la relation :

$$
n=\frac{M+\sqrt{M^{2}+4 n_{i}^{2}}}{2}
$$

avec

$$
M=N_{\mathrm{As}}+\frac{3}{2}\left(C_{\mathrm{As}}-N_{\mathrm{As}}\right)-C_{\mathrm{B}}
$$

qui assure la continuité avec la limite intrinsèque.

Dans MOBIDIC, seules les concentrations totales sont calculées, les dérivées des concentrations actives étant substituées à celles des concentrations totales selon la formule de différentiation suivante :

$$
\frac{\partial N_{\mathrm{As}}}{\partial x, y}=\frac{\partial N_{\mathrm{As}}}{\partial C_{\mathrm{As}}} \frac{\partial C_{\mathrm{As}}}{\partial x, y} .
$$

Suivant cette formulation, équations (4), (6) et (9), le couplage des impuretés est automatiquement pris en compte, la diffusion d'une impureté seule étant simplement réalisée en annulant la contribution de l'autre impureté.

La figure 3 illustre l'utilisation du modèle pour une simulation bidimensionnelle d'un prédépôt de bore dans lequel la concentration de surface $C_{\mathrm{s}}$ est maintenue constante. L'obtention d'un front de jonction abrupt caractérisé par le resserrement des isoconcentrations (Fig. 3a) et la forme du profil (Fig. 3b) est ici essentiellement dû à l'effet lacunaire [17]. Dans le cas de l'arsenic [19], figure 4, l'accélération de la diffusion est également présente, mais son effet est limité par la formation d'agglomérats. L'équilibre entre concentration totale et active, équation (9), est illustré sur le profil en coupe, figure $4 \mathrm{~b}$, et fait nettement apparaître la limite de solubilité de l'arsenic substitutionnel.

Pour le phosphore, un tout autre modèle est utilisé [20], qui rend compte de la forme caractéristique des profils observés expérimentalement. L'équation de diffusion de base s'écrit : 

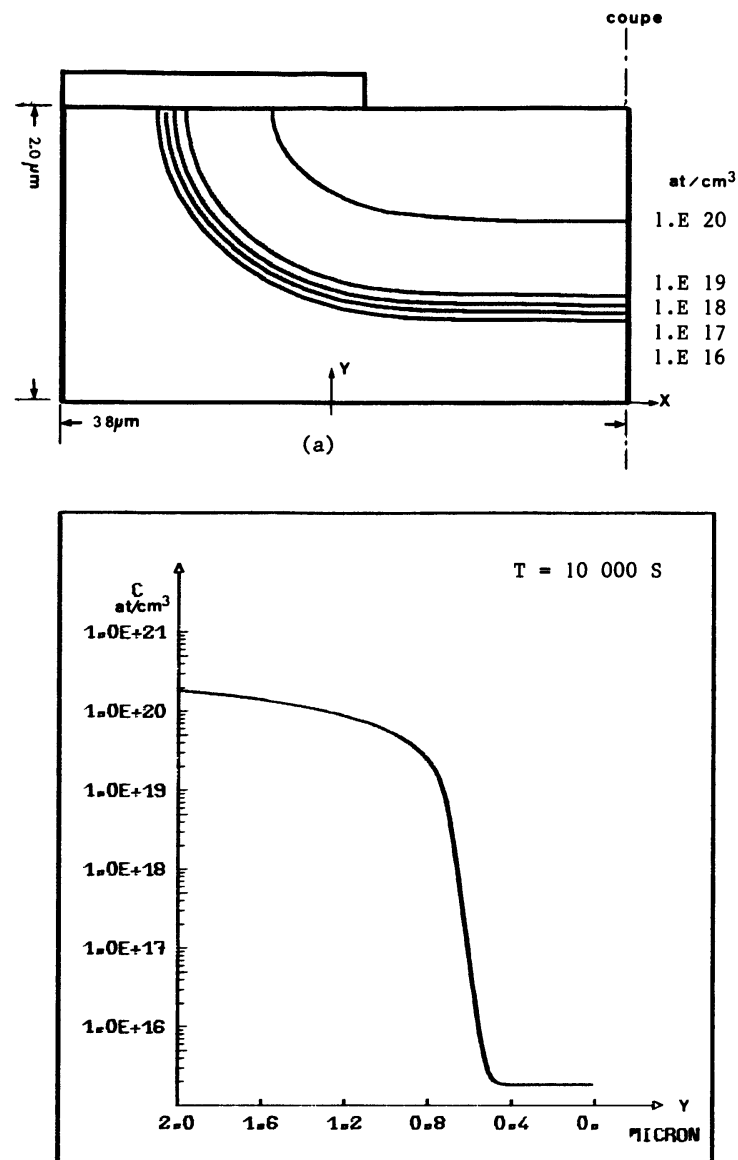

(b)

Fig. 3. - Simulation bidimensionnelle d'un prédépôt de bore : $D_{\mathrm{i}}=1 \times 10^{-14} \mathrm{~cm}^{-2} / \mathrm{s}, \quad n_{\mathrm{i}}=5 \times 10^{18} \mathrm{~cm}^{-3}$, $C_{\mathrm{s}}=1 \times 10^{20} \mathrm{~cm}^{-3}$. (a) Isoconcentrations $\left(\mathrm{cm}^{-3}\right)$; (b) Profil en coupe $(X=2 \mu \mathrm{m})$.

[Two dimensional simulation of a boron predeposition : $D_{\mathrm{i}}=1 \times 10^{-14} \mathrm{~cm}^{-2} / \mathrm{s}, n_{\mathrm{i}}=5 \times 10^{18} \mathrm{~cm}^{-3}, C_{\mathrm{s}}=1 \times$ $10^{20} \mathrm{~cm}^{-3}$. (a) Isovalues $\left(\mathrm{cm}^{-3}\right)$; (b) Cross-section view $(X=2 \mu \mathrm{m})$.]

$$
\frac{\partial C_{\mathrm{p}}}{\partial t}=\nabla \cdot\left[h \cdot D_{\mathrm{p}} \cdot \nabla C_{\mathrm{p}}\right]
$$

dans laquelle $C_{\mathrm{p}}$ est la concentration totale du phosphore, $D_{\mathrm{p}}$ son coefficient de diffusion effectif et $h$ le facteur d'accélération dû au champ électrique autoinduit :

$$
h=1+\frac{C_{\mathrm{p}} / 2 n_{\mathrm{i}}}{\sqrt{1+C_{\mathrm{p}}^{2} / 4 n_{\mathrm{i}}^{2}}} .
$$

Le coefficient de diffusion effectif, $D_{\mathrm{p}}$, présente trois plages de variation avec la concentration en phosphore :

- forte concentration :

$n \geqslant n_{\mathrm{e}}=4,61 \times 10^{21} \exp \left(-\frac{0,39 \mathrm{eV}}{k T}\right)$
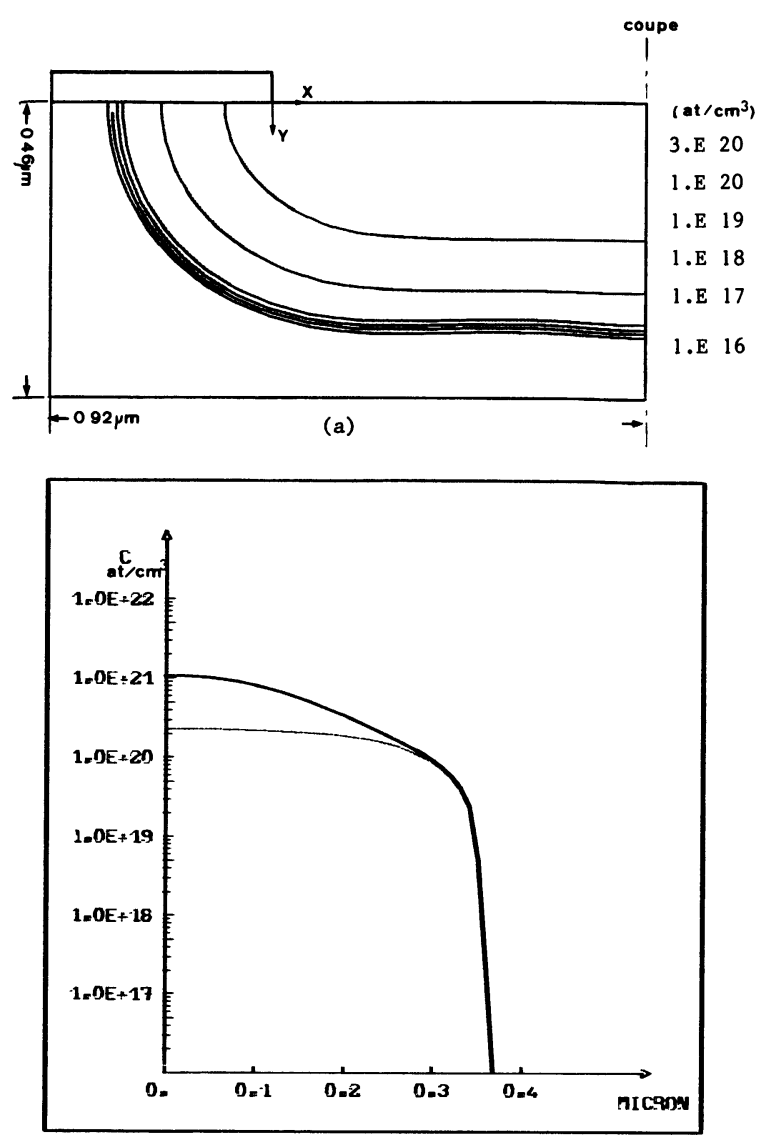

(b)

Fig. 4. - Recuit inerte $\left(1000^{\circ} \mathrm{C}, 20 \mathrm{~min}\right)$ d'arsenic implanté (140 keV, $\left.2 \times 10^{16} \mathrm{~cm}^{-2}\right)$. (a) Isoconcentrations $\left(\mathrm{cm}^{-3}\right)$; (b) Profil en coupe $(X=0,58 \mu \mathrm{m})$. Traits pleins : concentration atomique. Traits pointillés : concentration active.

[Inert annealing $\left(1000{ }^{\circ} \mathrm{C}, 20 \mathrm{~min}\right)$ of arsenic implantation $\left(140 \mathrm{keV}, 2 \times 10^{16} \mathrm{~cm}^{-2}\right)$. (a) Isovalues $\left(\mathrm{cm}^{-3}\right)$; (b) Crosssection view $(X=0.58 \mu \mathrm{m})$. Full lines : atomic concentration. Dashed lines : carrier concentration.]

$D_{1}=D^{\times}+D^{=}\left(\frac{n}{n_{\mathrm{i}}}\right)^{2} \cdot \exp \left(\frac{\Delta E g_{\mathrm{c}}+\Delta E g_{\text {i.o. }}}{k T}\right)$

- zone intermédiaire : $n<n_{\mathrm{e}}$

$D_{2}=\left[D^{\times}+D^{=}\left(\frac{n_{\mathrm{e}}}{n_{\mathrm{i}}}\right)^{2} \times\right.$

$\left.\exp \left(\frac{\Delta E g_{\mathrm{c}}+\Delta E g_{\text {i.o. }}}{k T}\right)\right]\left[\frac{n_{\mathrm{e}}}{n}\right]^{2}$,

- faible concentration : $D_{3} \geqslant D_{2}$

$$
\begin{array}{r}
D_{3}=D^{\times}+D^{-} \frac{n_{\max }^{3}}{n_{\mathrm{e}}^{2} n_{\mathrm{i}}}\left(1+\exp \frac{0,3 \mathrm{eV}}{k T}\right) \times \\
\exp \left(\frac{6 . \Delta E g_{\mathrm{c}}+3 . \Delta E g_{\text {i.o. }}}{2 k T}\right)
\end{array}
$$

$D^{\times}, D^{-}, D^{=}$sont les diffusivités liées aux divers types 
de lacunes. La concentration en électrons libres est calculée en fonction de la concentration en phosphore selon la relation suivante [20] :

$$
C_{\mathrm{p}}=n+2,04 \times 10^{-41} n^{3} .
$$

La zone de forte concentration correspond à une diffusion du phosphore par paires $\mathrm{P}^{+} \mathrm{V}^{=}$, tandis que dans la troisième zone, la diffusivité totale fait intervenir la contribution des paires $\mathrm{P}^{+} \mathrm{V}^{+}$, dont le degré de génération dépend de $n_{\max }$, la concentration maximale de porteurs libres présents dans la structure. Ce modèle tient également compte de la limitation de la diffusion du phosphore observée dans le cas de fort dopage. Cet effet est attribué à une réduction de largeur de bande interdite due aux contraintes introduites par les fortes concentrations de phosphore, $\Delta E g_{c}$, et aux dislocations créées par implantation ionique, $\Delta E g_{\text {i.o. }}$ [21]. Ces réductions de largeur de bande sont calculées à partir des valeurs instantanées de la concentration maximale en phosphore, $C_{\max }$ et de la dose d'implantation ionique $N_{\square}$ :

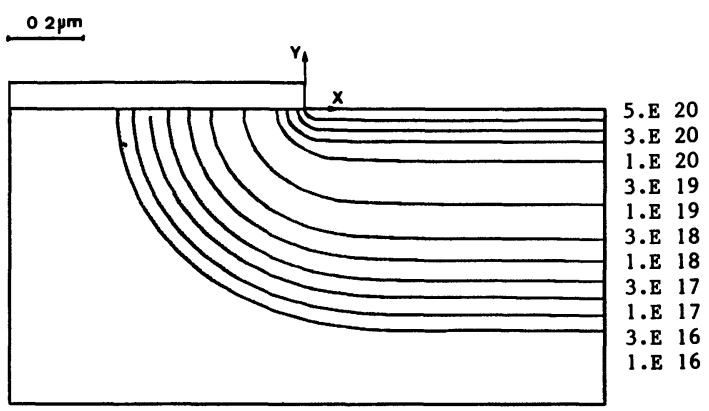

(a)

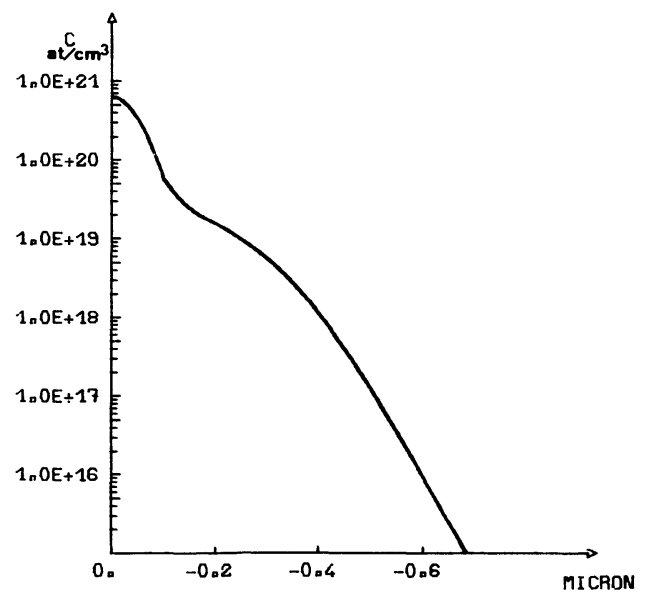

(b)

Fig. 5. - Simulation bidimensionnelle d'un prédépôt de phosphore : $C_{\mathrm{s}}=7 \times 10^{20} \mathrm{~cm}^{-3}, 30 \mathrm{~min}, 900^{\circ} \mathrm{C}$. (a) Isoconcentrations $\left(\mathrm{cm}^{-3}\right)$; (b) Profil en coupe $(X=0,8 \mu \mathrm{m})$.]

[Two dimensional simulation of a phosphorus predeposition : $C_{\mathrm{s}}=7 \times 10^{20} \mathrm{~cm}^{-3}, 30 \mathrm{~min}, 900^{\circ} \mathrm{C}$. (a) Isovalues $\left(\mathrm{cm}^{-3}\right)$; (b) Cross-section view $(X=0.8 \mu \mathrm{m})$.]

REVUE DE PHYSIQUE APPLIQÚE. - T. 21, № 1, JANVIER 1986

$$
\begin{aligned}
\Delta E g_{\mathrm{c}} & =-1,3 \times 10^{-22}\left(C_{\max }-C_{\text {seuil }}\right) \mathrm{eV} \\
\Delta E g_{\mathrm{i} . \mathrm{o}} & =-2,3 \times 10^{-6}\left(N_{\square}\right)^{0,25} \mathrm{eV} .
\end{aligned}
$$

$C_{\text {seuil }}$ étant le seuil de prise en compte de $\Delta E g_{c}$, dépendant de la température.

La figure 5 a présente sous forme d'isovaleurs les résultats d'une simulation bidimensionnelle d'un prédépôt de phosphore.

La représentation en coupe de ce résultat, figure $5 b$, illustre les effets du modèle utilisé notamment la formation de la zone de plateau et l'apparition d'un point d'inflexion dans le profil.

$\mathrm{Vu}$ le manque actuel de modèles validés décrivant le couplage du phosphore avec les autres impuretés, le couplage dans MOBIDIC est empiriquement réalisé par une extension des modèles précédents avec une concentration en électrons libres, $n$, obtenue par une somme quadratique des équations (9) et (14).

2.3 OXYDATION DU SILICIUM MONOCRISTALLIN. - La simulation de l'oxydation du silicium doit être incluse dans les possibilités d'un simulateur opérationnel de technologie. En effet, la plupart des étapes de recuit s'effectuent en ambiance oxydante, pour laquelle l'interaction diffusion-oxydation est très importante. La simulation de l'oxydation doit principalement s'intéresser à la cinétique de croissance d'oxyde et à la ségrégation des impuretés à l'interface $\mathrm{Si} / \mathrm{SiO}_{2}$.

Une méthode analytique est utilisée pour simuler la croissance bidimensionnelle d'oxyde. Dans le cas d'une oxydation de champ (procédé LOCOS), la variation de l'épaisseur d'oxyde en fonction de la dimension latérale $d_{\mathrm{ox}}(x, t)$ est simulée par [22] :

$$
\begin{aligned}
d_{\mathrm{ox}}(x, t)=d_{\mathrm{i}}+ & \left(d_{\mathrm{ox}_{1 \mathrm{D}}}(t)-d_{\mathrm{i}}\right) \times \\
\frac{1}{2} & {\left[1+\operatorname{erf}\left(\frac{\sqrt{2}}{\gamma} \cdot \frac{x}{d_{\mathrm{ox}_{1 \mathrm{D}}}(t)-d_{\mathrm{i}}}\right)\right] }
\end{aligned}
$$

dans laquelle $d_{\mathrm{i}}$ est l'épaisseur uniforme d'oxyde initial, $d_{\mathrm{ox}_{1 \mathrm{D}}}$, l'épaisseur d'oxyde à 1 dimension, donnée par la loi parabolique de Deal-Grove [23]. Le paramètre $\gamma$, qui caractérise l'extension de l'oxydation latérale est calculé à partir des conditions expérimentales [24]. Le profil d'oxyde est schématisé en figure 6, l'origine des abscisses étant déterminée par la lithographie du nitrure de silicium avant oxydation.

Lors d'une phase d'oxydation du silicium dopé, la redistribution des impuretés à l'interface $\mathrm{Si} / \mathrm{SiO}_{2}$ conserve la continuité des potentiels chimiques de chaque dopant dans les deux matériaux. Cette condition d'équilibre crée une discontinuité du profil de concentration à la frontière $\mathrm{Si} / \mathrm{SiO}_{2}$ qui est caractérisée par le coefficient de ségrégation $m$, défini par :

$$
m=\frac{C_{\mathrm{Si}}}{C_{\mathrm{SiO}_{2}}}
$$

où $C_{\mathrm{Si}}$ et $C_{\mathrm{SiO}_{2}}$ sont respectivement la concentration 


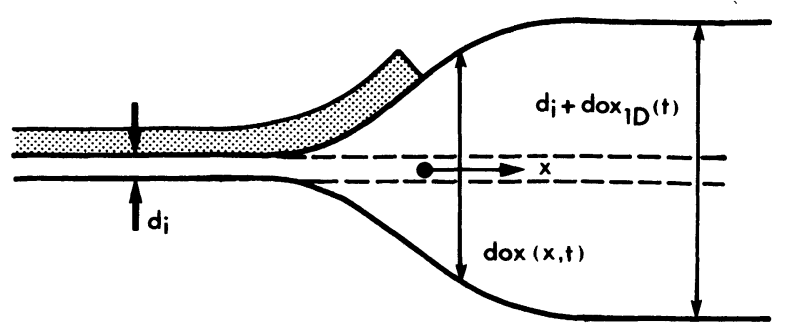

Fig. 6. - Forme bidimensionnelle d'oxyde.

[Two-dimensional oxide shape.]

à l'interface côté silicium et côté silice. Pour le bore le coefficient de ségrégation est à l'équilibre calculé en fonction de la température et de l'orientation du silicium, [1] tandis que pour l'arsenic et le phosphore il est pris constant $(m=10)$ [1].

De nombreux résultats expérimentaux ont montré que la diffusivité du bore et du phosphore est accélérée sous ambiance oxydante. Ce phénomène(O.E.D.) est attribué à une diffusion induite par des atomes de silicium interstitiel générés à l'interface $\mathrm{Si} / \mathrm{SiO}_{2}$. Sous ambiance oxydante la diffusivité du bore et du phosphore est modifiée selon la relation suivante [25] :

$D_{\text {ox }}=D+F \cdot V_{\text {ox }}^{0,3} \exp \left(-\frac{2,07 \mathrm{eV}}{k T}\right) \cdot \exp \left(-\frac{Y}{2 \mu m}\right)$

où $V_{\text {ox }}$ est la vitesse de croissance d'oxyde, $F$ une constante dépendant du type de dopant et de l'orientation du silicium. Le terme $\exp (-Y / 2 \mu m)$ rend compte de l'atténuation latérale de l'effet d'accélération de la diffusion dans le cas d'oxydation localisée. Il prend effet sous le masque de nitrure, $Y$ est la distance latérale comptée depuis le bord du masque [26].

\section{Méthode numérique.}

Hormis le cas de l'implantation ionique, la simulation des autres étapes technologiques nécessite la résolution d'équations différentielles partielles afin d'obtenir l'évolution spatiale et temporelle de la distribution des dopants. Dans MOBIDIC, la discrétisation spatiale est de type éléments finis. La résolution temporelle est menée par une procédure itérative.

\subsection{DisCrétisation SPatiale et TEMPORELle. - La} méthode consiste ici à décomposer le domaine en éléments simples (triangles 6 noeuds), reliés entre eux par les nouds du maillage. Les inconnues du problème sont les valeurs nodales des concentrations. Les équations sont traitées suivant une technique classique de résidus pondérés du type Galerkin [27]. La solution est restituée sur tout le domaine en utilisant les fonctions de pondération propres à chaque élément. Le principal avantage d'une discrétisation de type éléments finis réside dans le fait que chaque élément est topologiquement indépendant. Ceci permet de reproduire fidèlement des formes de surface quelconque et d'exploiter des raffinements locaux de maillage afin de réduire le nombre total de noeuds de calcul et donc les temps d'exécution [28].

L'écriture des équations de diffusion dans le formalisme éléments finis [29] conduit à un système matriciel

$$
[K(\mathbf{C})] \mathbf{C}+[M] \dot{\mathbf{C}}=0
$$

dans lequel $\mathbf{C}$ et $\dot{\mathbf{C}}$ sont respectivement les vecteurs formés par les valeurs des concentrations et de leurs dérivées temporelles aux nouds du domaine $[K(C)]$ et $[M]$ sont respectivement les matrices de diffusion et de masse du système discrétisé.

La résolution itérative du système matriciel de l'équation (19) est réalisée par un schéma temporel implicite incomplet :

$$
[M] \frac{\mathbf{C}_{n+1}-\mathbf{C}_{n}}{\Delta T}+\left[K\left(\mathbf{C}_{n}\right)\right] \mathbf{C}_{n+1}=0
$$

dans lequel $\Delta T$ est lincrément de temps, choisi constant et $n$ le numéro de boucle. Cette méthode permet de n'utiliser qu'un seul niveau de boucle, mais a nécessité une étude systématique du choix de l'incrément de temps afin de s'affranchir des effets numériques inhérents aux différents modèles de diffusion [30].

3.2 Simulation du COUPLAGe ENTRe IMPURETÉs. Dans le schéma numérique utilisé, deux méthodes peuvent être envisagées afin de simuler la diffusion couplée de deux impuretés : l'intégration simultanée du système complet $(20)$, ou la résolution alternée de chaque équation de diffusion. La seconde solution a été retenue qui préserve le conditionnement et la symétrie des matrices. De plus, cette méthode augmente le degré d'implicité de la résolution temporelle tout en limitant les temps de calcul et l'espace mémoire requis. Un exemple de diffusion couplée est présenté en figure 7. Le tracé d'isovaleur et le profil de coupe attestent de la prise en compte correcte du couplage bore-arsenic dans cet exemple technologique qui est une extension à 2 dimensions d'un résultat unidimensionnel classique [31].

3. 3 Simulation De l'oXYdation. - Différentes solutions ont déjà été proposées pour résoudre les équations de diffusion dans un domaine évolutif tout en tenant compte de la ségrégation à l'interface $\mathrm{Si} / \mathrm{SiO}_{2}$. Les techniques les plus couramment utilisées sont des méthodes de transformation ponctuelle $[4,5,9]$ pour lesquelles les équations sont résolues dans un domaine stationnaire ou des méthodes d'élimination de nouds [7].

Comme le code éléments finis, MOBIDIC, permet des déplacements arbitraires des points de discréti- 

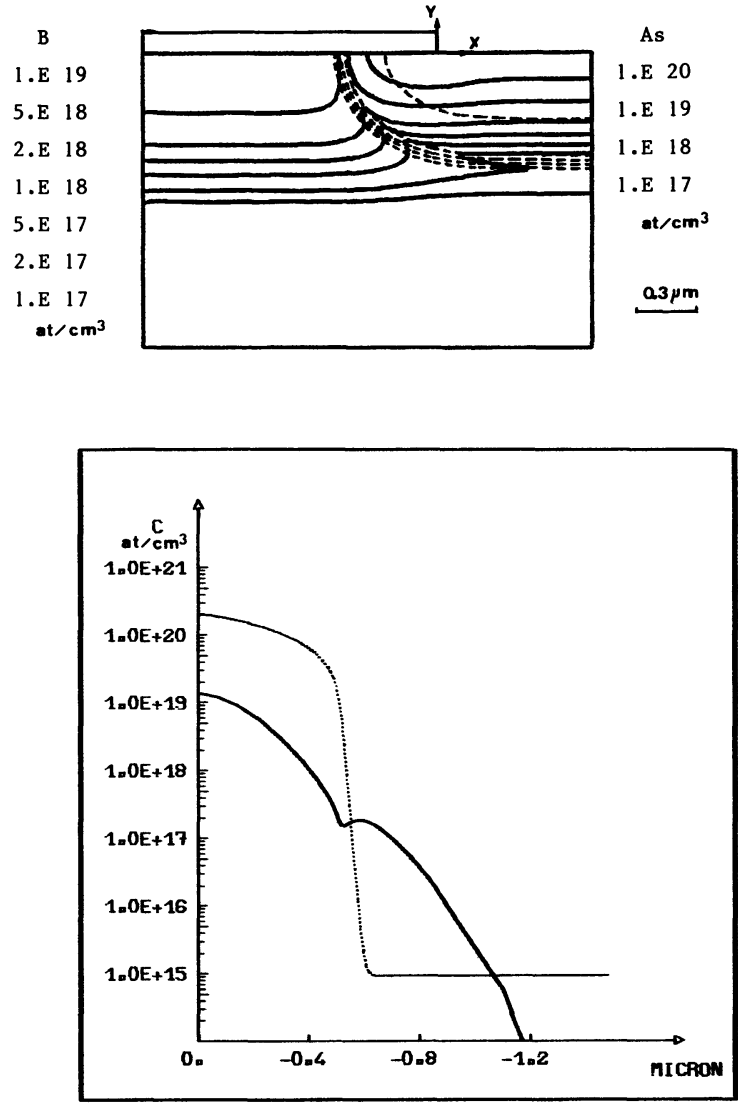

Fig. 7. - Simulation bidimensionnelle d'une structure NPN. - Prédépôt uniforme de bore : $D_{\mathrm{i}}=1,2 \times 10^{-13} \mathrm{~cm}^{2} \mathrm{~s}^{-1}$, $C_{\mathrm{s}}=2,27 \times 10^{19} \mathrm{~cm}^{-3}, \quad T=1200 \mathrm{~s}$ (fonction erfc). - Prédépôt local d'arsenic : $D_{1}=1,2 \times 10^{-14} \mathrm{~cm}^{2} \mathrm{~s}^{-1}$, $C_{\mathrm{s}}=2,27 \times 10^{20} \mathrm{~cm}^{-3}, n_{\mathrm{i}}=5,68 \times 10^{18} \mathrm{~cm}^{-3}, T=1500 \mathrm{~s}$. (a) Isoconcentrations; (b) Profil en coupe ( $X=0,78 \mu \mathrm{m})$. Traits pleins : bore $\left(\mathrm{cm}^{-3}\right)$. Traits pointillés : arsenic.

[Two-dimensional simulation of a NPN structure. Uniform boron predeposition : $D_{1}=1.2 \times 10^{-13} \mathrm{~cm}^{2} \mathrm{~s}^{-1}, C_{\mathrm{s}}=$ $2.27 \times 10^{19} \mathrm{~cm}^{-3}, T=1200 \mathrm{~s}$ (erfc function). Local arsenic predeposition : $D_{1}=1.2 \times 10^{-14} \mathrm{~cm}^{2} \mathrm{~s}^{-1}, C_{\mathrm{s}}=2.27$ $\times 10^{20} \mathrm{~cm}^{-3}, n_{\mathrm{i}}=5.68 \times 10^{18} \mathrm{~cm}^{-3}, T=1500 \mathrm{~s}$. (a) Isovalues $\left(\mathrm{cm}^{-3}\right)$; (b) Cross-section view $(X=0.78 \mu \mathrm{m})$; Full lines : boron; Dashed lines : arsenic.]

sation, les équations de diffusion sous ambiance oxydante sont écrites sans changement de variable. La procédure consiste en l'alternance d'étapes de diffusion, équation (16), et d'étapes de déformation de maillage.

Durant la phase de diffusion, les équations sont résolues uniquement dans le silicium, les effets de croissance et de ségrégation étant contenus dans une condition de Neuman imposée à l'interface oxyde/ silicium [5] :

$$
F=-V_{\text {ox }} C_{\mathrm{Si}}\left(\frac{1}{m}-\alpha\right)
$$

dans laquelle $V_{\text {ox }}$ est la vitesse normale d'oxydation locale, $m$ le coefficient de ségrégation et $\alpha$ le rapport volumétrique de la réaction d'oxydation $(\alpha=0,44)$. Ce flux inclut, outre le mécanisme de ségrégation, l'effet de déformation du maillage et vérifie la conservation de la dose totale dans le système silice/silicium.

L'étape de remaillage inclut la déformation de la structure conformément à l'équation (16), et une procédure d'interpolation permettant la copie des cartes de dopage sur les maillages successifs.

Cette méthode a été appliquée à la simulation d'un procédé LOCOS dans lequel l'implantation de bore, auto-alignée par le masque de nitrure est redistribuée sous ambiance oxydante humide [4]. La figure 8 présente le profil de bore à l'issue du recuit.

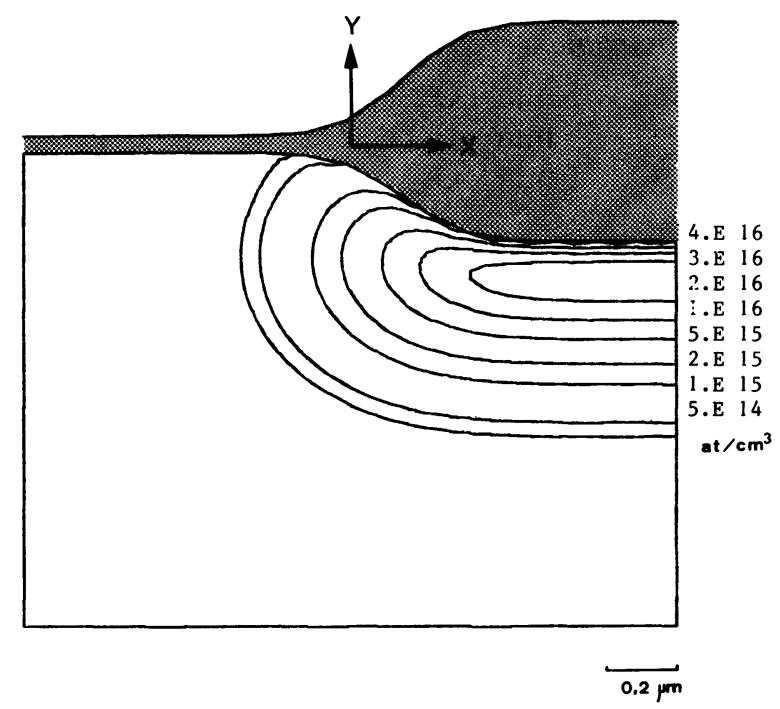

Fig. 8. - Simulation d'un procédé LOCÓS. Implantation de bore : $120 \mathrm{keV}, N_{\mathrm{a}}=2,3 \times 10^{12} \mathrm{~cm}^{-2}, d_{\mathrm{i}}=0,05 \mu \mathrm{m}$. Recuit sous $\mathrm{H}_{2} \mathrm{O}, 1000{ }^{\circ} \mathrm{C}, 120 \mathrm{~min}$. Isoconcentration de bore en $\mathrm{cm}^{-3}$.

[LOCOS process simulation. Boron implantation $120 \mathrm{keV}$, $N_{\mathrm{a}}=2.3 \times 10^{12} \mathrm{~cm}^{-2} ; d_{\mathrm{i}}=0,05 \mu \mathrm{m}$. Annealing $\mathrm{H}_{2} \mathrm{O}$, $1000{ }^{\circ} \mathrm{C}, 120 \mathrm{~min}$. Boron isovalues in $\mathrm{cm}^{-3}$.]

Ce résultat illustre l'effet de la ségrégation sur la redistribution du bore et permet de définir l'extension latérale des zones de champ.

\section{Couplage simulation de technologie-simulation de dispositif pour une technologie n-MOS.}

Le calcul de profils bidimensionnels de dopage présente non seulement un intérêt technologique direct, mais permet de plus, une bonne description topologique du transistor indispensable à la simulation de dispositif. L'exploitation du couplage entre un simulateur de technologie et un simulateur de dispositif (couplage technologie/dispositif) s'avère être un outil performant pour la mise au point des enchaînements technologiques. Il permet numériquement la détermination des compromis de fabrication [32] et 
l'obtention des sensibilités de comportements électriques aux paramètres technologiques.

Un tel système est en cours de tests. Une interface a été réalisée entre les programmes MOBIDIC et MINIMOS 2.2 [2] et le couplage de ces deux logiciels est étudié dans le cas d'une technologie n-MOS 6 microns-grille polysilicium.

4.1 Simulation DU DOPAGE SOURCE/DRAIN. - Le procédé technologique concernant le dopage et les recuits des zones de source et drain peut se décomposer en 3 phases :

a) Dopage.

Implantation phosphore $120 \mathrm{keV}, 5 \times 10^{15} \mathrm{~cm}^{-2}$.

b) Recuit ambiance oxydante.

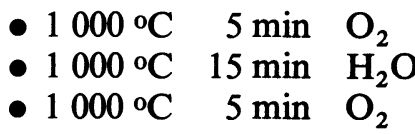

c) Recuit ambiance inerte.

- $1000^{\circ} \mathrm{C} 10 \mathrm{~min} \mathrm{~N}_{2}$

- $1000^{\circ} \mathrm{C} 15 \mathrm{~min}$

- L'implantation ionique est réalisée à travers $950 \AA$ Å d'oxyde de grille sur une structure auto-alignée, le masque étant déterminé par le profil d'attaque de la grille polycristalline. Un relevé précis de la structure de masquage avant implantation a été réalisé afin de calculer la fonction de masque nécessaire au calcul du profil, équation (3). Une étude de reproductibilité a également été menée qui a conduit au profil d'implantation illustré en figure 9.

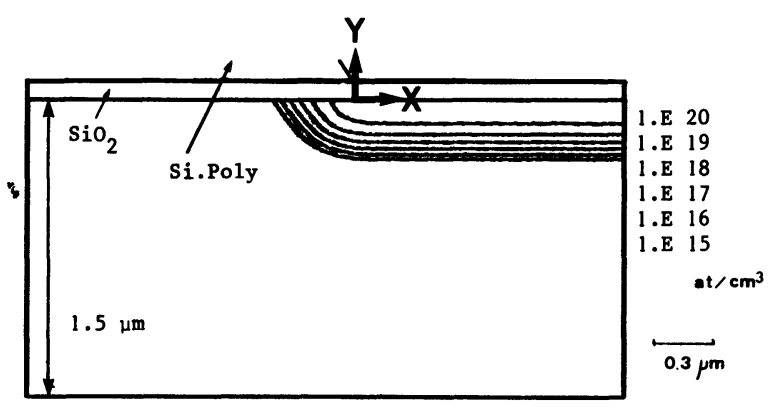

Fig. 9. - Profil de dopage après l'implantation ionique (phase a). Isoconcentrations.

[Doping profile after the implantation (a-step). Isovalues.]

- La simulation des recuits en ambiance oxydante a nécessité un relevé du profil final d'oxyde. $\mathrm{Ce}$ contrôle a permis de constater que la loi de croissance bidimensionnelle d'oxyde de champ, équation (16) peut être également appliquée pour caractériser l'interface $\mathrm{Si} / \mathrm{SiO}_{2}$ sur les structures de source et drain $(\gamma=1,7)$. Ce relevé donne également la réduction de la longueur de grille polycristalline due à son oxydation latérale. L'origine du système, extrémité du matériau polycristallin en fin de fabrication, est alors déterminée. Elle permet de définir la diffusion latérale et la longueur de grille électrique pour la simulation de dispositif. Des motifs de coloration [26] ont également été réalisés, qui ont prouvé la validité de l'expression du coefficient de diffusion en ambiance oxydante, équation (19), dans le procédé présent. La figure 10 présente le profil simulé à l'issue des 3 étapes d'oxydation, la forme des isoconcentrations près de l'interface illustrant l'effet de la ségrégation.

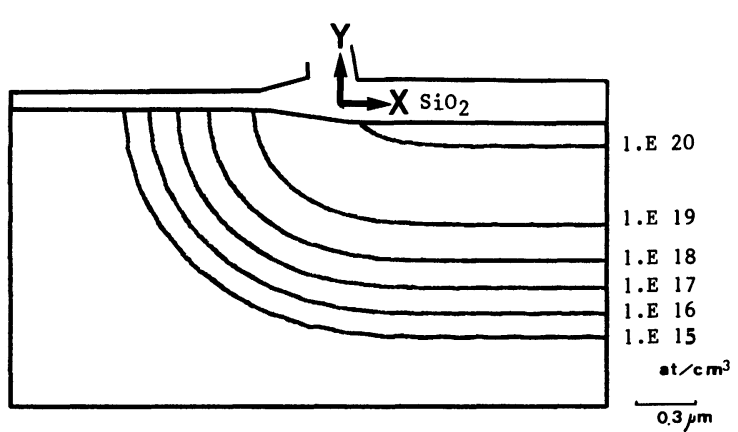

Fig. 10. - Profil de dopage après les recuits oxydants (phase b). Isoconcentrations.

[Doping profile after the oxidizing ambient annealing (bstep). Isovalues.]

- La troisième phase technologique, correspondant aux étapes haute température intervenant en fin de fabrication, a été simulée en ambiance inerte. Le profil final, figure 11, donne la jonction bidimensionnelle en fin de procédé et démontre ici une diffusion latérale importante.

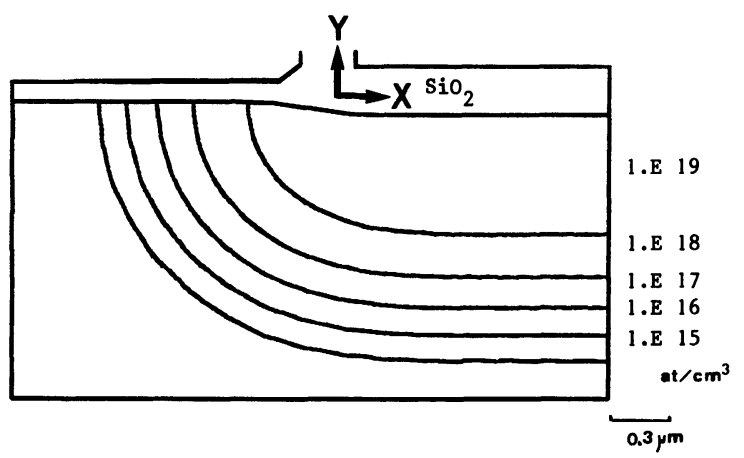

Fig. 11. - Profil de dopage en fin de procédé (phase c). Isoconcentrations.

[Doping profile at the end of the process (c-step). Isovalues.]

4.2 INTERFACE TECHNOLOGIE-DISPOSITIF. - La description physique complète d'un transistor MOS est obtenue par des opérations de symétrie et de translation du profil de concentration source-drain. 
Ces opérations géométriques sont réalisées automatiquement en fonction de la longueur de grille électrique. Afin de réduire au maximum les temps de calcul nécessaires à la simulation de technologie, les profils de concentration dans le canal, obtenus par une simulation typiquement unidimensionnelle, sont fournis par le programme SUPREM II [1]. La figure 12 présente la structure complète d'un transistor réalisé dans la technologie 6-microns. La simulation du dispositif nécessite un fichier d'entrée de type MINIMOS 2.2 [2] dans lequel sont spécifiques les 4 tensions de polarisation (substrat, source, grille, drain) ainsi que l'épaisseur d'oxyde de grille et la densité de charge à l'interface $\mathrm{Si} / \mathrm{SiO}_{2}$. A titre d'exemple, les figures $13 \mathrm{a}$ et $13 \mathrm{~b}$, présentent respectivement le tracé d'équipotentiels et la distribution de la densité latérale du courant pour le transistor de la figure 12. Ces figures correspondent à une longueur de grille de $5 \mathrm{~m} \mu$ et à une polarisation définie comme suit :

$$
\begin{array}{ll}
\text { Tension substrat } & =0 \\
\text { Tension source } & =0 \\
\text { Tension grille } & =2 \mathrm{~V} \\
\text { Tension drain } & =5 \mathrm{~V} .
\end{array}
$$

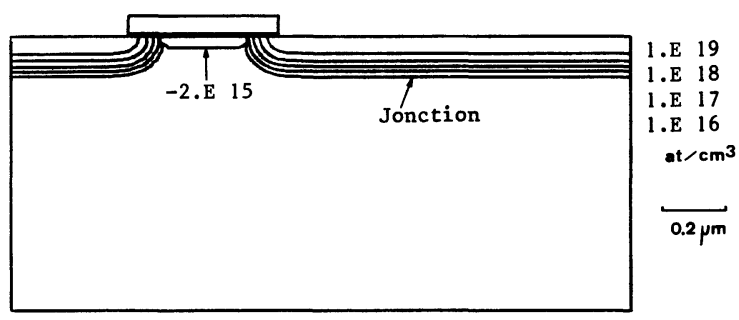

Fig. 12. - Profil de dopage complet d'un transistor réalisé dans la technologie n-MOS, $L_{\text {grille }}=5 \mu \mathrm{m}$.

[Complete doping profile of a transistor realised in the $\mathrm{n}$ MOS process, $L_{\text {gate }}=5 \mu \mathrm{m}$.]

La démarche qui va être maintenant suivie vise à l'obtention de confrontation simulation-expérimentation au niveau électrique sur le comportement statique des transistors. Cette étude est en cours de réalisation, mais son degré d'avancement ne permet pas encore la présentation de résultats quantitatifs.

\section{Conclusion.}

Sur une base numérique de type éléments finis, un simulateur bidimensionnel de technologie a été développé. La souplesse des algorithmes utilisés permet de résoudre les différents modèles d'implantation ionique, de diffusion et d'oxydation relatifs au procédé de fabrication de circuits intégrés silicium. Un effort particulier a été porté au niveau du choix des modèles et de leur intégration afin de reproduire avec précision les effets importants dus à la réduction des dimensions, tels que le couplage entre impuretés, la diffusion de l'arsenic sous forte concentration et la ségrégation des impuretés à l'interface $\mathrm{Si} / \mathrm{SiO}_{2}$.

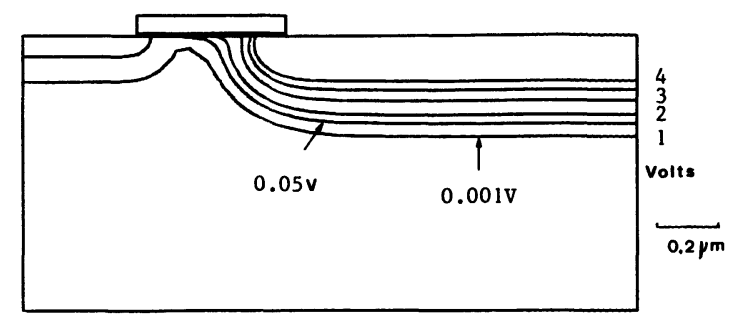

a)

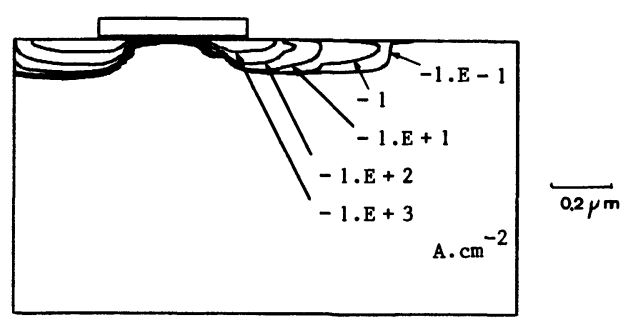

b)

Fig. 13. - Résultats de la simulation de dispositif sur le transistor de la figure 12. $V_{\mathrm{S}}=V_{\mathrm{B}}=0 ; V_{\mathrm{G}}=2 \mathrm{~V} ; V_{\mathrm{D}}=$ 5 V. (a) Equipotentiels $(V)$. (b) Densité latérale de courant (A. $\mathrm{cm}^{-2}$ ).

[Device simulation results for the transistor plotted in figure 12. $V_{\mathrm{S}}=V_{\mathrm{B}}=0 ; V_{\mathrm{G}}=2 \mathrm{~V} ; V_{\mathrm{D}}=5 \mathrm{~V}$. (a) Electric potential (V). (b) Lateral current density $\left(\mathrm{A} . \mathrm{cm}^{-2}\right)$.]

Une interface avec le programme MINIMOS a été rendu opérationnelle. Elle permet d'introduire les résultats de la simulation de technologie dans un simulateur de dispositif afin d'avoir accès aux informations concernant le comportement électrique des structures actives. Les performances potentielles d'un tel système sont d'un très grand intérêt. En effet, elles permettent d'entrevoir, de manière purement numérique, l'optimisation des séquences technologiques et la détermination de la sensibilité des performances électriques des transistors aux paramètres de fabrication.

Le travail qui a été ici présenté s'oriente vers trois axes de développement. Le premier concerne la simulation et l'optimisation de procédé industriel avec le programme, MOBIDIC, dans sa version actuelle, conformément aux objectifs du GCIS. Dans un second temps des tests précis du couplage technologie-dispositif seront menés sur quelques technologies éprouvées afin d'obtenir des confrontations au niveau électrique.

Enfin, les possibilités de la simulation bidimensionnelle de technologie seront étendues vers la modélisation du silicium polycristallin pour des raisons liées à son utilisation croissante dans les techniques d'intégration.

\section{Remerciements.}

Ce travail a été réalisé avec l'appui du Groupement Circuit Intégré Silicium (G.C.I.S.). Les auteurs remercient M. E. Caquot et M. S. Martin (C.N.E.T., C.N.S.) pour les discussions fructueuses qu'ils ont eues avec eux tout au long de cette étude. 
Bibliographie

[1] Antoniadis, D. A. et Dutton, R. W., I.E.E.E. J. Solid State Circuits SC 14 (1979) 412.

[2] Selberherr, S., Schutz, A. et Potzl, H. W., I.E.E.E. Trans. Electron. Devices ED 27 (1980) 1540.

[3] Greenfield, J. A. et Dutton, R. W., I.E.E.E. Trans. Electron. Devices ED 27 (1980) 1520.

[4] TIELERT, R., N.A.T.O. A.S.I. on process and Devices Simulation for MOS-VLSI circuits, Urbino, Italy (July 1982).

[5] Penumalli, B. R., I.E.E.E. Trans. Electron. Devices ED 30 (1983) 986

[6] Chin, D., LeE, H. G. et Dutton, R. W., I.E.E.E. Trans. Electron. Devices ED 29 (1982) 336.

[7] TANigushi, K., Kashiwagi, M. et IwaI, H., I.E.E.E. Trans. Electron. Devices ED 28 (1981) 574.

[8] Caquot, E. et Marocco, A., Proceed. 2nd Int. Conf. on numerical Analysis of Semiconductor Devices and I.C.'S Dublin, Ireland (June 1981).

[9] Maldonado, C. D., Custode, F. Z., Louie, S. A. et PANCHOLY, R., I.E.E.E. Trans. Electron. Devices ED 30 (1983) 1462.

[10] Salsburg, K. A. et Hansen, H. H., I.E.E.E. Trans. Electron. Devices ED 30 (1983) 1004.

[11] Runge, H., Phys. Status Solidi A 39 (1977) 3770.

[12] Gibbons, H. F., Johnson, W. S. et Mylroie, S. W., Projected Range Statistics (Stroudsburg, Ed. Dowdon, Hutchinson, Ross) 1975.

[13] Gibbons, J. F. et Mylroie, S., Appl. Phys. Lett. 222 (1973) 568.

[14] Hofker, W. F., Phillips Res. Repts, Suppl. No 8 (1975).

[15] Ryssel, H., Haberger, K., Hoffmann, K., Prinke, G., DuMCK, R. et SACHS, A., I.E.E.E. Trans. Electron. Devices ED 27 (1980) 1484.

[16] Hu, S. M., Phys. Rev. 180 (1969) 773.
[17] Warner, D. D. et Wilson, C. L., BSTJ 59 (1980) 1.

[18] Guerrero, E., Potzl, H., Tielert, R., GrasserBAUER, M. et Stingeder, G., J. Electrochem. Soc. 129 (1982) 1826.

[19] Tsai, M. Y., Morehead, F. F., Baglin, J. E. E. et Michel, A. E., J. Appl. Phys. 51 (1980) 3230.

[20] FaIR, R. B. et Tsai, J. C. C., J. Electrochem. Soc. 124 (1977) 1107.

[21] FaIR, R. B., J. Appl. Phys. 50 (1979) 860.

[22] Penumalli, B. R., Proceed. 2nd Int. Conf. on Numerical Analysis of Semiconductor Device and I.C.'S, Dublin, Ireland (June 1981).

[23] Deal, B. E. et Grove, A. S., J. Appl. Phys. 36 (1965) 3770.

[24] Wu, T. C., Stacy, W. T. et Ritz, N. N., J. Electrochem. Soc. 130 (1983) 1563.

[25] Tanigushi, K., Kurosawa, K. et Kashiwagi, M., J. Electrochem. Soc. 127 (1980) 2243.

[26] Lin, A. M., Dutton, R. W. et Antoniadis, D. A., Appl. Phys. Lett. 35 (1977) 799.

[27] ZIENkIEWICZ, O. C., The finite element method (McGraw-Hill, New York) 1977.

[28] Collard, D. et Decarpigny, J. N., 3rd Int. Conf. on Numerical Analysis of Semiconductor Device and I.C.'S Galway, Ireland (June 1983).

[29] Collard, D. et Decarpigny, J. N., Simulation bidimensionnelle de processus de diffusion, rapport G CIS (1981).

[30] Collard, D. et Decarpigny, J. N., COMPEL 1 (1984) 17.

[31] Hu, S. M. et Schmidt, S., J. Appl. Phys. 39 (1968) 4272.

[32] Iwai, H., Tanigushi, K., Konaka, M., Maeda, S. et Nishi, Y., I.E.E.E. Trans. Electron. Devices ED 29 (1982) 625. 